\title{
Differential Relative Sulfation of Keratan Sulfate Glycosaminoglycan in the Chick Cornea during Embryonic Development
}

\author{
Melody Liles, ${ }^{1}$ Barbara P. Palka, ${ }^{1}$ Anthony Harris, ${ }^{2}$ Briedgeen Kerr, ${ }^{2}$ Clare Hughes, ${ }^{2}$ \\ Robert D. Young, ${ }^{1}$ Keith M. Meek, ${ }^{1}$ Bruce Caterson, ${ }^{2}$ and Andrew J. Quantock ${ }^{1}$
}

\begin{abstract}
Purpose. To investigate structural remodeling of the developing corneal stroma concomitant with changing sulfation patterns of keratan sulfate (KS) glycosaminoglycan (GAG) epitopes during embryogenesis and the onset of corneal transparency.
\end{abstract}

Methods. Developing chick corneas were obtained from embryonic day (E)12 to E18 of incubation. Extracellular matrix composition and collagen fibril spacing were evaluated by synchrotron $\mathrm{x}$-ray diffraction, hydroxyproline assay, ELISA (with antibodies against lesser and more highly sulfated KS), and transmission electron microscopy with specific proteoglycan staining.

Results. A significant relative increase in highly sulfated KS epitope labeling occurred with respect to hydroxyproline content in the final week of chick development, as mean collagen interfibrillar distance decreased. Small KS PG filaments increased in frequency with development and were predominantly fibril associated.

Conclusions. The accumulation of highly sulfated KS during the E12 to E18 timeframe could serve to fine tune local matrix hydration and collagen fibril spacing during corneal growth, as gross dehydration and compaction of the stroma progress through the action of the nascent endothelial pump. (Invest Ophthalmol Vis Sci. 2010;51:1365-1372) DOI:10.1167/iovs.09-4004

$\mathrm{T}$ he cornea is a connective tissue with remarkable transparency that forms the primary refractive surface of the eye; yet, how this property arises in development remains little understood. The most widely studied model of corneal development is that of the embryonic chicken. ${ }^{1,2}$ In this tissue, uniformly thin collagen fibrils are laid down by stromal fibroblasts in a secondary stroma and consolidate as a series of orthogonal fibril bundles that later form into lamellae. ${ }^{3}$ At

From the ${ }^{1}$ Structural Biophysics Group, School of Optometry and Vision Sciences, and the ${ }^{2}$ Connective Tissue Biology Laboratories, School of Biosciences, Cardiff University, Cardiff, Wales, United Kingdom.

Supported by Grant EP/F034970/1 from the Engineering and Physical Sciences Research Council (AJQ, RDY, ML, KMM), Grant BBS/B/ 10994 from the Biotechnology and Biological Sciences Research Council (AJQ, BC), Grant G0001033 (KMM, AJQ) and Grant MRC G0800248 (AH, BK, CH, BC) from the Medical Research Council, UK, and Grant ARC 17540 from the Arthritis Research Campaign (AH, BK, CH, BC).

Submitted for publication May 19, 2009; revised July 10 and August 7, 2009; accepted August 7, 2009.

Disclosure: M. Liles, None; B.P. Palka, None; A. Harris, None; B. Kerr, None; C. Hughes, None; R.D. Young, None; K.M. Meek, None; B. Caterson, None; A.J. Quantock, None

Corresponding author: Andrew J. Quantock, Structural Biophysics Group, School of Optometry and Vision Sciences, Cardiff University, Maindy Road, Cardiff CF24 4LU, UK; quantockaj@cf.ac.uk. embryonic day (E)14, the chick cornea transmits only approximately $40 \%$ of white light, but then begins to increase in transparency so that, at E19, transmission is more than 95\%, similar to the adult condition. ${ }^{4}$ The transparency increase after E14 is accompanied by significant dehydration of the cornea, flattening of keratocytes, and compaction of stromal collagen fibrils into a spatially ordered array. ${ }^{5,6}$ This restructuring of the stroma is key for the acquisition of corneal transparency. ${ }^{7,8}$

Small leucine-rich proteoglycans (PGs) interact with collagen fibrils in the corneal stroma and are thought to help control fibril size and spatial organization. ${ }^{9}$ PGs are composed of a core protein covalently bound to sulfated glycosaminoglycan (GAG) side chains. The major GAG in the cornea is keratan sulfate (KS), and three types of PG bear these side chains: lumican, ${ }^{10}$ keratocan, ${ }^{11}$ and mimecan (or osteoglycin). ${ }^{12}$ By E18, in the chick stroma KS PGs bear two to three GAG chains of approximately $15 \mathrm{kDa}$ each, ${ }^{13}$ and the PGs lumican and keratocan have three of five potential linkage sites variably substituted with KS GAGs. ${ }^{14}$ Collagen-PG interactions are thought to occur via the PG core protein, with sulfated GAG chains extending into the interfibrillar space, where they confer stromal water-binding and sorptive tendencies that help define the hydrophilicity and swelling properties, and thus the ultrastructure, of the stromal matrix. ${ }^{15}$

Corneal PGs, especially those carrying KS chains, fulfill an important role in the establishment of a properly formed stroma during corneal embryogenesis. ${ }^{16}$ The importance of these molecules is illustrated by the fact that the corruption of sulfate motifs on KS is associated with structural matrix alterations in the corneas of humans with the inherited disease macular corneal dystrophy ${ }^{17,18}$ and in the corneas of mice with gene-targeted deletions in lumican ${ }^{19,20}$ or Chst5, a KS sulfotransferase enzyme. ${ }^{21}$ Some investigators have reported the accumulation of KS GAGs and KS PGs during embryonic development. $^{22-25}$ Molecular studies have shown that levels of mRNA for keratocan, first expressed at E6, ${ }^{26}$ exhibit a steady decline from E9 to E18, whereas, over the same time period, lumican mRNA levels remain several-fold higher. ${ }^{27}$ Moreover, an approximate threefold increase in lumican core protein occurs between E7 and E9, ${ }^{28}$ with a two- to threefold increase in mRNA for $\beta$-1,4-galactosyltransferase, an enzyme involved in sulfated KS synthesis, reported between E8 and E13. ${ }^{29}$ Biochemical analyses have also shown that KS is synthesized by the chick cornea between E5 and E7, but that it only becomes highly sulfated by $\mathrm{E} 14,{ }^{30}$ with another study indicating a switch in the production of unsulfated to sulfated KS between $\mathrm{E} 12$ and E15. ${ }^{28}$

The current investigation was designed to test the hypothesis that sulfation of KS provides an environment of hydration conducive to the deposition of collagen fibrils and the establishment of a highly ordered stromal matrix in the developing chick. To this end, experiments were conducted to quantify 
collagen biosynthesis, KS sulfation status, collagen fibril spacing, and collagen-KS PG associations during the latter stages of chick corneal morphogenesis.

\section{Methods}

\section{Specimens}

For x-ray diffraction and biochemical studies, a series of 69 embryonic chick corneas was obtained that comprised daily incremental stages from E12 to E18. Corneas were excised by an incision around the limbus in eyes of embryonic chicks obtained from Hamburger-Hamilton (HH)-staged, fertilized eggs collected from a commercial hatchery (Hy-Line UK, Warwickshire, UK) approximately 2 hours earlier, where $\mathrm{E} 12=\mathrm{HH} 38, \mathrm{E} 13=\mathrm{HH} 39, \mathrm{E} 14=\mathrm{HH} 40, \mathrm{E} 15=\mathrm{HH} 11, \mathrm{E} 16=\mathrm{HH} 42$, $\mathrm{E} 17=\mathrm{HH} 43$ and E18 $=$ HH44. Immediately on excision, the corneas were sandwiched between layers of clingfilm to minimize dehydration and were placed on dry ice. Frozen corneas were transferred to $-80^{\circ} \mathrm{C}$ storage until the synchrotron $\mathrm{x}$-ray diffraction experiments could be conducted. For electron microscopy, a smaller group of fertilized eggs (Henry Stewart \& Co. Ltd., Louth, Lincolnshire, UK) was incubated at $37^{\circ} \mathrm{C}$ until staging and excision. All work was conducted in accordance with the ARVO Statement for the Use of Animals in Ophthalmic and Vision Research, as well as with local ethics guidelines.

\section{Synchrotron X-Ray Diffraction}

Individual corneas ( $n=69)$, still secured in cling film, were positioned in a sealed specimen holder between sheets of x-ray-transparent mylar where they were allowed to thaw. In turn, each cornea $(n=8-11$ daily from E12 to E18) was then placed into the path of a focused $(1 \times$ $1 \mathrm{~mm})$ monochromatic $(\lambda=0.154 \mathrm{~nm}) \mathrm{x}$-ray beam on beamline 2.1 of the Synchrotron Radiation Source, Daresbury Laboratory (Cheshire, UK). All corneas were exposed for 2 minutes each, and the resulting fiber diffraction patterns were recorded on a multiwire, gas proportional area detector plate positioned $8.25 \mathrm{~m}$ behind the cornea. Immediately after $x$-ray exposure, the corneas were refrozen and transported to the home laboratories for biochemical and immunochemical analyses (described in the following sections). Diffraction patterns $(512 \times$ 512 pixels) were analyzed with purpose-written software (Unix-based; Sun Microsystems, Mountain View, CA) and graphics and statistics packages (Unix; Optimas, Bothell, WA; Statistica; Statsoft, Tulsa, OK, Excel; Microsoft, Redmond, WA), as described previously. ${ }^{31}$ The position of the first-order equatorial (i.e., interfibrillar) reflection, calibrated to the $67-\mathrm{nm}$ axial D-periodic repeat of collagen in wet rat tail tendon, was used to provide a measure of the mean center-to-center collagen fibril Bragg spacing as an average throughout the whole thickness of the central cornea. Statistical significance was ascertained by using statistical tests for large data groups: one-way ANOVA with post-hoc Tukey HSD.

After $\mathrm{x}$-ray analysis, the corneas were refrozen for transportation to the home laboratory and digested for 17 hours at $60^{\circ} \mathrm{C}$ with $1 \mathrm{mg} / \mathrm{mL}$ papain (Sigma-Aldrich, Poole, UK) in $0.05 \mathrm{M}$ sodium acetate buffer ( $\mathrm{pH}$ 5.6), containing $0.025 \mathrm{M}$ EDTA and $5 \mathrm{mM}$ cysteine $\mathrm{HCl}$. The enzyme was inactivated at $100^{\circ} \mathrm{C}$, and the digests stored at $-20^{\circ} \mathrm{C}$ until further analysis.

\section{Hydroxyproline Assay}

Papain digests from individual corneas were hydrolyzed by using equal volumes of $11.7 \mathrm{~N}$ concentration $\mathrm{HCl}$ to supernatant at $110^{\circ} \mathrm{C}$ overnight. Specimens were then lyophilized. Dried hydrolyzates were reconstituted in their starting volume of distilled water and centrifuged to remove particulate material. Hydroxyproline residues were assayed in triplicate as previously described, ${ }^{32}$ against known standards and read at $540 \mathrm{~nm}$ after 10 to 20 minutes incubation at $70^{\circ} \mathrm{C}$. Statistical significance was ascertained by using statistical tests for large data groups: one-way ANOVA with post-hoc Tukey HSD.

\section{KS Quantification by Competitive ELISA}

Optimized, competitive ELISAs were developed to quantify pentasulfated hexasaccharides and tetrasulfated hexasaccharides as the smallest linear structures in KS chains, as recognized by the monoclonal antibodies $5 \mathrm{D} 4$ and $1 \mathrm{~B} 4$, respectively. ${ }^{33-35}$ Ninety-six-well EIA microtiter plates (MP Biomedicals, Cambridge, UK) were coated by passive adsorption with a $250 \mathrm{ng} / \mathrm{mL}$ chondroitinase $\mathrm{ABC}$-digested bovine articular cartilage aggrecan (BAC ABC core) antigen in a $20-\mathrm{mM}$ sodium carbonate coating buffer ( $\mathrm{pH} 9.6$ ), for 14 hours at $37^{\circ} \mathrm{C}$. Native aggrecan core protein is substituted with both chondroitin sulfate (CS) and KS chains, and CS was selectively removed by chondroitinase digestion to leave a KS-linked coating protein. The plates were washed with Tris saline azide (TSA) and the unreacted sites blocked with the addition of $1 \%$ (wt/vol) bovine serum albumin (BSA) in TSA. All incubations were performed for 1 hour at $37^{\circ} \mathrm{C}$.

Papain digests from single corneas were serially diluted and allowed to bind with an equal volume of 5D4 (1:8000 dilution in 1\% BSA/TSA) and incubated to compete against the BAC ABC core. A standard curve was generated from serial dilutions of BAC ABC core/ 5D4. The plates were then washed with TSA before incubation with alkaline phosphatase-conjugated goat anti-mouse antibody (1:5000 dilution; Promega, Madison, WI). The plates were again washed before alkaline phosphatase substrate (p-nitrophenyl phosphate, $1 \mathrm{mg} / \mathrm{mL}$ ) was applied in DEA buffer $(0.126 \mathrm{mM} \mathrm{MgCl}, 1 \mathrm{M}$ diethanolamine, $\mathrm{pH}$ corrected to 9.8). Color development was quantified on a plate reader (Multiskan MS; Labsystems, Helsinki, Finland) at $405 \mathrm{~nm}$, to determine the inhibition of binding.

The same stock corneal extracts were then assayed for lesser sulfated KS by using the 1-B- 4 monoclonal antibody. Optimization of conditions established that microtiter plates were coated with 125 $\mathrm{ng} / \mathrm{mL} \mathrm{BAC} \mathrm{ABC}$ core and ELISAs performed as described above, with a 1:4000 1-B-4 dilution. Statistical significance was ascertained with statistical tests for large data groups: one-way ANOVA with post-hoc Tukey HSD.

\section{Electron Microscopy}

On excision, corneas from E12 to E18 chicks $(n=21)$ were prepared for electron microscopy, as described previously. ${ }^{21,36}$ A series of E12 to E18 corneas was fixed in $2.5 \%$ glutaraldehyde in $25 \mathrm{mM}$ sodium acetate buffer with $0.05 \%$ cuprolinic blue $\left(0.1 \mathrm{M} \mathrm{MgCl}_{2} ; \mathrm{pH} 5.7\right)$, to stain the sulfated PGs. Another series was fixed for 10 minutes in $4 \%$ paraformaldehyde ( $\mathrm{pH}$ 7.2). These corneas were then washed twice in chondroitinase buffer $(50 \mathrm{mM}$ Tris, $[\mathrm{pH} 8], 60 \mathrm{mM}$ sodium acetate, and $0.02 \% \mathrm{BSA}$ ) and quartered. Corneal pieces were incubated for 4 hours at $37^{\circ} \mathrm{C}$ in chondroitinase buffer containing $2.5 \mathrm{U} / \mathrm{mL}$ chondroitinase ABC (from Proteus vulgaris; Sigma-Aldrich) to remove the chondroitin- and dermatan-sulfate GAG chains from the tissue. Commercially prepared protease inhibitor cocktail for general use (SigmaAldrich) was also added to $1 \%$ total volume to inhibit endogenous PG-degradative activity. The control corneas were similarly processed in buffer minus enzyme. The samples were washed in chondroitinase buffer (minus enzyme) before fixation and staining overnight, as before. All corneas were then contrast enhanced with sodium tungstate and dehydrated and embedded in Araldite, according to standard protocols. ${ }^{36}$ Ultrathin sections $(\sim 90$-nm thick) were cut on glass knives, collected on copper grids, and stained with $1 \%$ aqueous phosphotungstic acid and saturated aqueous uranyl acetate before examination by transmission electron microscopy (JEM-1010; JEOL, Tokyo, Japan) equipped with an 11-megapixel CCD camera (Orius SC1000 CCD camera; Gatan Ltd., UK, Cambridge, UK).

\section{Results}

Mesenchymally derived presumptive keratocytes that populate the developing chick cornea are responsible for establishing the secondary stroma by synthesizing and depositing hybrid collagen type I/V fibrils. ${ }^{1,2}$ Quantification of collagen as hy- 
TABLE 1. Average Amount of Hydroxyproline and Average Collagen Interfibrillar Spacing in Embryonic Chick Corneas

\begin{tabular}{|c|c|c|c|c|c|c|c|}
\hline & $\begin{array}{c}\mathrm{E} 12 \\
(n=8)\end{array}$ & $\begin{array}{c}\mathrm{E} 13 \\
(n=10)\end{array}$ & $\begin{array}{c}\text { E14 } \\
(n=11)\end{array}$ & $\begin{array}{c}\mathrm{E} 15 \\
(n=10)\end{array}$ & $\begin{array}{c}\mathrm{E} 16 \\
(n=11)\end{array}$ & $\begin{array}{c}\mathrm{E} 17 \\
(n=10)\end{array}$ & $\begin{array}{c}\mathbf{E 1 8} \\
(n=9)\end{array}$ \\
\hline Hydroxyproline, $\mu \mathrm{g} / \mathrm{mL}$ & $4.38 \pm 0.18$ & $8.06 \pm 1.07$ & $7.43 \pm 0.48$ & $17.48 \pm 1.46$ & $17.23 \pm 0.74$ & $34.52 \pm 4.11$ & $34.69 \pm 1.64$ \\
\hline $\begin{array}{l}\text { Collagen fibril } \\
\text { Bragg spacing, nm }\end{array}$ & $60.8 \pm 0.60$ & $63.1 \pm 1.01$ & $63.9 \pm 0.99$ & $61.9 \pm 1.22$ & $59.8 \pm 1.53$ & $57.0 \pm 0.87$ & $53.7 \pm 0.94$ \\
\hline
\end{tabular}

Embryonic days E12 to E18 are equivalent to Hamburger-Hamilton stages 38 to 44 of development. Hydroxyproline measurements are also representative of total collagen content per cornea. Data are expressed as the mean $\pm \mathrm{SE}$.

droxyproline (Table 1) indicated two significant increases in hydroxyproline content, found between E14 and E15 $(P<$ $0.005)$ and E16 and E17 $(P=0.001)$. At E16 Coleman et al. ${ }^{37}$ measured $20.7 \mu \mathrm{g}$ hydroxyproline per cornea and Conrad, ${ }^{38}$ $19.5 \mu \mathrm{g}$. Our value of $17.23 \mu \mathrm{g} /$ cornea at this developmental stage is consistent with these published values.

$\mathrm{X}$-ray diffraction patterns generated by corneas at E12 gave rise to collagen interfibrillar reflections that were consistent with a rather loose, hydrated network of formative lamellae that constitutes the tissue at this time. With development, interfibrillar reflections became more intense, presumably as a consequence of growth and the added deposition of collagen as fibrils, consistent with the results of the hydroxyproline analysis shown in Table 1 . Analysis of all 69 x-ray diffraction patterns showed that the average center-to-center collagen fibril spacing from E14 to E18 decreased as the cornea thinned and became more transparent (Table 1).

These collagen spacing data were highly representative of the corneal stroma as a whole, with all fibrils in the path of the x-ray beam (which measured approximately $1 \times 1 \mathrm{~mm}$ ) passing through the whole thickness of the cornea contributing to the $\mathrm{X}$-ray pattern and thus the calculated values. X-ray analysis, however, could not identify how the stromal matrix was changing at the suprafibrillar level. Electron micrographs confirmed the overall nature of the stromal compaction and indicated, not a homogeneous coming together of widely and uniformly spaced fibrils with time, but rather a progressive coalescence of bundles of collagen fibrils in which the fibrils were already fairly well organized and packed laterally (Fig. 1). Based on the fundamental principles of x-ray fiber diffraction, the collagen-free spaces between fibril bundles, which were quite prevalent before E15, did not contribute to collagen interfibrillar reflection. Thus, the values displayed in Table 1 derive from collagen fibrils that are regularly spaced, whether they exist in bundles, as in the earlier stages that we investigated, or in stacked lamellae, as seen later in development. Our observations (Fig. 1), together with those of other investigators $^{3,6}$ showed that stromal compaction during development entailed collagen fibril bundles coming together to form lamellae, whereas $\mathrm{X}$-ray data indicated that fibrils within the bundles and lamellae are moving closer together from E14 onward.

As synchrotron $\mathrm{x}$-ray diffraction is a noninvasive technique, the 69 corneas from which ultrastructural collagen fibril data were acquired (Table 1) were available for subsequent immunochemical quantification of KS epitope. Data were normalized to hydroxyproline content to reflect changes in lesser- sulfated (1B4-immunoreactive) and highly sulfated (5D4immunoreactive) KS epitope, relative to matrix deposition (Table 2). It is important to remember that these values represent relative epitope labeling between samples and that the nature of the antibodies used potentially allows multiple binding sites within the same GAG chain. KS values are relative to $\mathrm{BAC}-\mathrm{ABC}$ core protein labeling equivalents.

Levels of highly sulfated KS as a proportion of hydroxyproline throughout the E12-to-E18 interval were consistently higher than corresponding amounts of the low-sulfated epitope. The amount of lesser-sulfated KS fluctuated between E12 and E18, but did not change appreciably, whereas levels of highly sulfated KS increased markedly. As can be seen in Table 2, highly sulfated KS levels remained fairly steady between E13 and $\mathrm{E} 15$, after which a sharp rise occurred $(P=0.001)$, such that by E16 the average amount had more than doubled from levels at E15. Although there was a reduction subsequently, it is not statistically significant $(P=0.528)$. Examination by electron microscopy of corneas in the E12-to-E18 timeframe in which chondroitin/dermatan sulfate GAG chains had been enzymatically removed from the tissue revealed cuprolinic bluecontrasted sulfated KS PGs as small collagen fibril-associated filaments that increased in abundance with time.

Discrete fibril bundles coalescing into a continuous matrix have been observed by other investigators ${ }^{3}$ and are apparent in Figures 1, 2. Measurements of (1) hydroxyproline, (2) highly and lesser sulfated KS, and (3) collagen fibril spacing were performed for each of the 69 corneas examined (Tables 1, 2). Consequently, we can seek correlations independent of developmental day. In doing so, it is evident that a positive correlation exists between hydroxyproline content and highly sulfated KS, as assessed by 5D4 binding $\left(R^{2}=0.682\right)$, and between hydroxyproline content and lesser-sulfated KS, as measured by $1 \mathrm{~B} 4$ binding $\left(R^{2}=0.395\right)$, as shown in Figure 3 . These parallel changes presumably reflect embryonic growth and continued matrix deposition. However, no appreciable correlation was found between the ratio of KS to hydroxyproline and collagen fibril spacing, when data from all 69 corneas were analyzed independent of developmental stage (Fig. 4).

\section{Discussion}

The changing sulfation patterns of KS in the developing chick cornea raise several questions regarding the likely functional role of this molecule with respect to its sulfation status. Sulfa-

TABLE 2. The Average Amounts of 1B4- and 5D4-Labeled KS Epitope Relative to BAC-ABC Core after Normalization to Hydroxyproline Content in Embryonic Chick Corneas

\begin{tabular}{|c|c|c|c|c|c|c|c|}
\hline & $\operatorname{E12}(n=8)$ & $\operatorname{E13}(n=10)$ & $\operatorname{E14}(n=11)$ & $\operatorname{E15}(n=10)$ & $\operatorname{E16}(n=11)$ & $\mathrm{E} 17(n=10)$ & $\operatorname{E18}(n=9)$ \\
\hline $\begin{array}{l}\text { Lesser sulfated KS (1B4-labeled } \\
\text { epitope/hydroxyproline) }\end{array}$ & $0.125 \pm 0.043$ & $0.128 \pm 0.032$ & $0.153 \pm 0.046$ & $0.129 \pm 0.024$ & $0.109 \pm 0.016$ & $0.114 \pm 0.022$ & $0.118 \pm 0.024$ \\
\hline $\begin{array}{l}\text { Highly sulfated KS (5D4-labeled } \\
\text { epitope/hydroxyproline) }\end{array}$ & $0.775 \pm 0.261$ & $0.958 \pm 0.152$ & $0.865 \pm 0.160$ & $0.840 \pm 0.111$ & $1.879 \pm 0.167$ & $1.449 \pm 0.143$ & $1.874 \pm 0.206$ \\
\hline
\end{tabular}



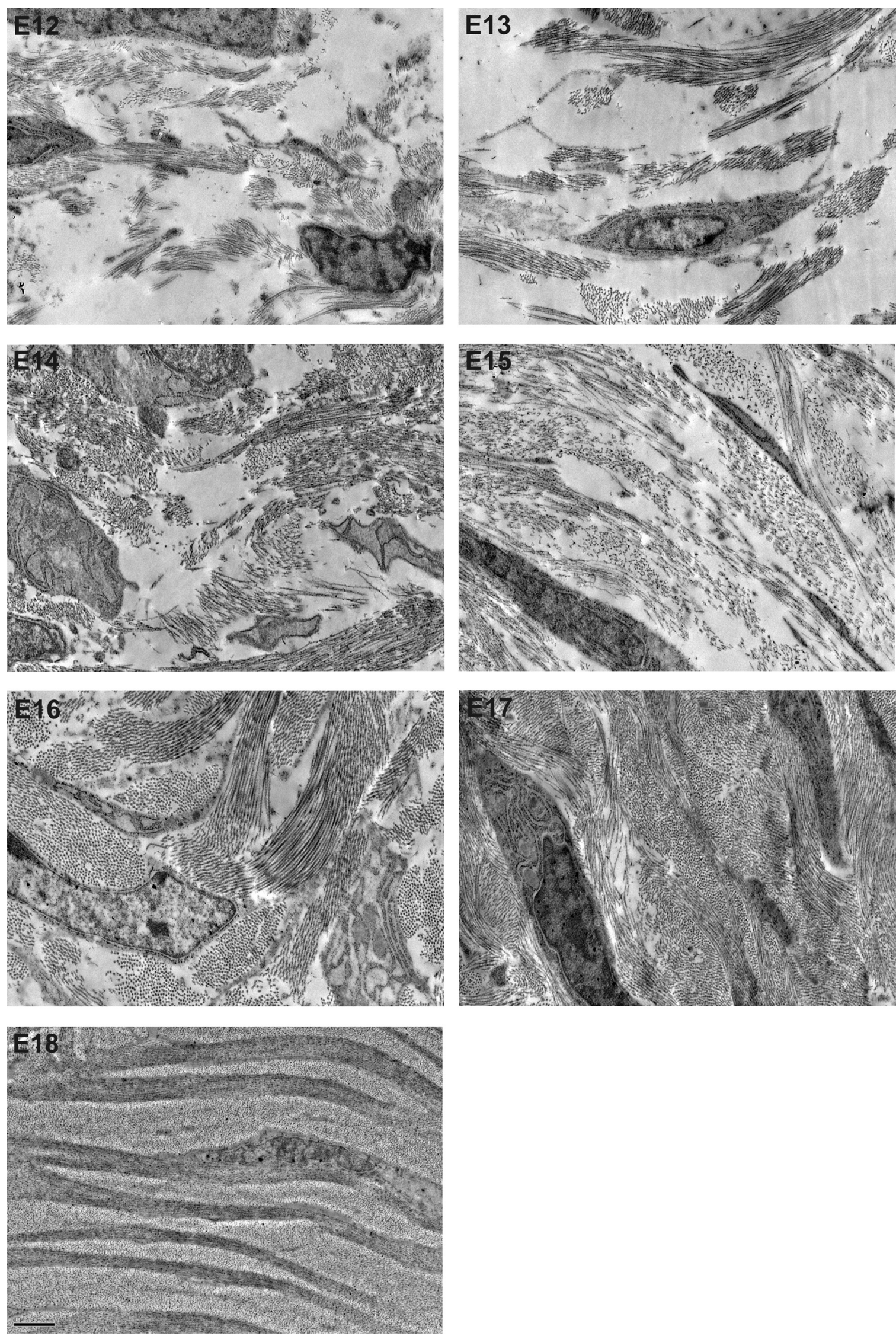

FigURE 1. Transmission electron micrographs of developing chick corneal stroma. Tissue compaction progressed from loose bundles of collagen fibrils at E12 to a mature lamellar form at E18. Scale bar, $1 \mu \mathrm{m}$ 

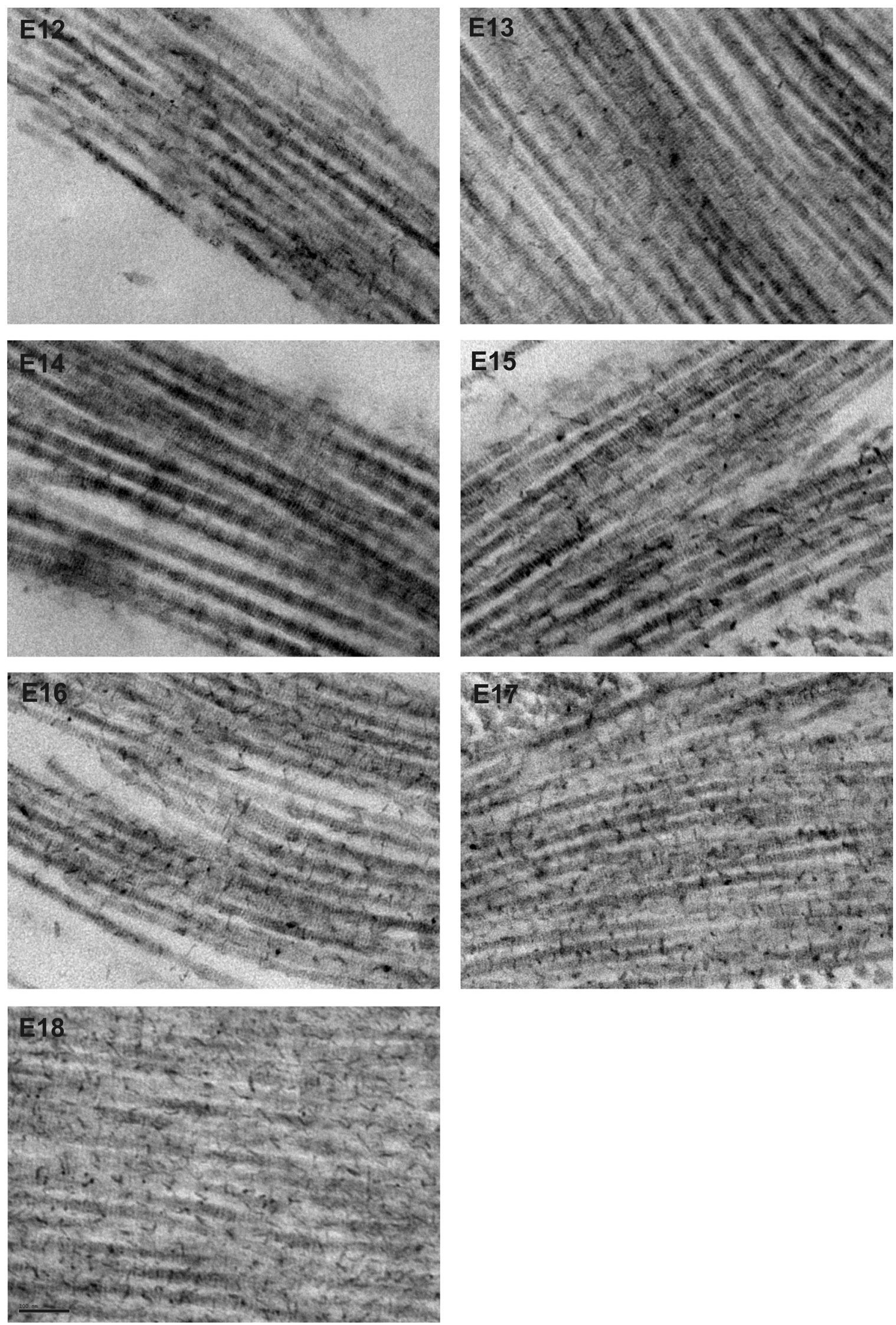

FIGURE 2. Transmission electron micrographs of chondroitinase digested developing chick corneal stroma. Increased deposition of cuprolinic blue-stained sulfated KS PG filaments occurs in the E12-E18 timeframe. Scale bar, $1 \mu \mathrm{m}$. 


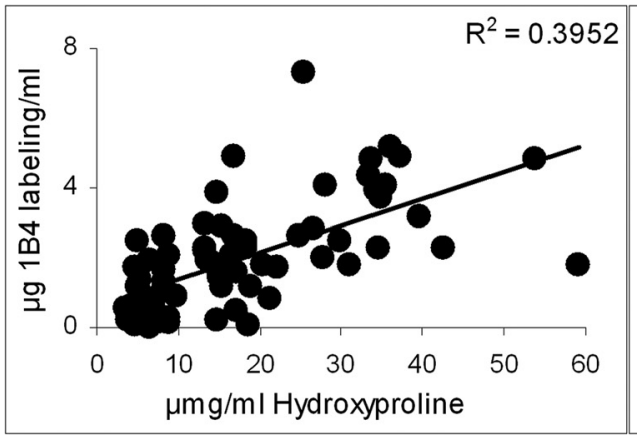

Figure 3. Correlation of antibody labeling in relation to BAC-ABC core against hydroxyproline content (without normalization to hydroxyproline, raw data not shown) for lesser (left, $1 \mathrm{~B} 4$ reactive) and more highly (right, 5D4 reactive) sulfated $\mathrm{KS}$ tion of individual corneal KS chains is not homogeneous, and unsulfated disaccharides, which are predominant nearer the linkage region, become increasingly sulfated toward the nonreducing terminal. ${ }^{39}$ Moreover, KS chains undergo sequential elongation and sulfation during synthesis. ${ }^{40}$ The absence of sulfotransferase enzyme in human corneas with macular corneal dystrophy, as in mutant corneas of Chst5-knockout mice, results in an immature, truncated GAG and an atypical corneal phenotype. ${ }^{18,21}$ Thus, KS chain length and sulfation are dictated by tissue-specific factors such as the presence of processing enzymes. During chick development, galactose-transferase activity increases with $\mathrm{KS}$ biosynthesis and is maintained at an unusually high level in adult cells. ${ }^{29}$ The sequential mechanism of GAG subunit sulfation becomes apparent in the absence of the sulfotransferase enzyme, when neither GlcNAc nor Gal residues are sulfated. ${ }^{41}$ The negative charge provided by sulfation could serve to prevent KS GAG chain collapse during elongation and perhaps offer an explanation of the differential sulfation between shorter and longer KS chains. The presence of increasing amounts of highly sulfated KS during embryonic development is possibly linked to the appearance of the sulfate donor, 3'-phosphoadenosine- 5 '-phosphosulfate (PAPS). ${ }^{42}$ The measured elevation of 5D4 labeling at the time of increased fibrillogenesis and matrix compaction could be a result of PAPS availability facilitating increases in KS chain length and greater sulfation. The combination of these events could enhance organized stroma formation through the binding of core proteins and the hydrophilic influence of sulfate groups.

A major purpose of the present study was to investigate the structural remodeling of the stroma in relation to changing sulfation patterns of constitutive KS epitope during chick corneal morphogenesis. The results confirm previous synchrotron $\mathrm{x}$-ray diffraction studies that documented the progressive compaction of corneal collagen fibrils with embryonic growth in the chick. ${ }^{43,44}$ In the dataset examined herein, compaction occurred after E14 (Table 1), with electron microscopy indicating that the compaction happened within bundles of collagen fibrils in which fibrils are already fairly well organized spatially (Fig. 1). The x-ray diffraction and electron microscopy findings, when considered together, imply that collagen bundles are coalescing into lamellae, whereas, at the same time, fibrils within bundles are becoming more closely spaced. Between E15 and E16, the stroma experiences a significant increase in highly sulfated KS epitope that is disproportionate to the measured increase in hydroxyproline (Table 2). This observation is illustrative of an accelerated deposition of highly sulfated KS post-E15 which exceeds the anticipated in ovo corneal growth rate, as implied by hydroxyproline deposition. Thus, as the chick cornea develops and becomes transparent and compacted, the nature of KS antigenicity changes, with the highly sulfated KS fraction accumulating preferentially over that of the lesser-sulfated isoform and from a higher starting point. It seems counterintuitive to reason that negatively charged, highly sulfated KS GAG would accumulate as the stroma condenses and act as a biological driving force for tissue dehydration. Quite the opposite: The water-retentive properties of this hydrophilic macromolecule would be expected to swell the corneal matrix, rather than to aid in its compaction. Indeed, the current data show that the stroma was already undergoing compaction when the cornea, at E15, experienced the first sizable increase in highly sulfated KS epitope.

This result suggests that the emergence of more highly sulfated KS GAG in the chick cornea as it matures in ovo is not a driving force correlating with the homogeneous compaction of collagen fibrils in the coalescing collagen bundles and developing stromal lamellae and perhaps points to the influence of other modulating factors that help drive the structural remodeling of the developing corneal stroma in the week before hatching.

Activity of the bicarbonate pump in the corneal endothelium is critical for controlling corneal hydration and thickness and thus for maintaining transparency in the adult eye. ${ }^{45-47}$ It is also likely to be important in stromal water regulation and compaction in the developing cornea. However, the precise stage at which the endothelial pump mechanism becomes active is unknown. Histologic investigations have shown that an intact endothelial monolayer is established by $\mathrm{E} 5$ to $\mathrm{E} 6 .^{5}$ Stromal fluid at this time is mostly immobilized by hyaluronic
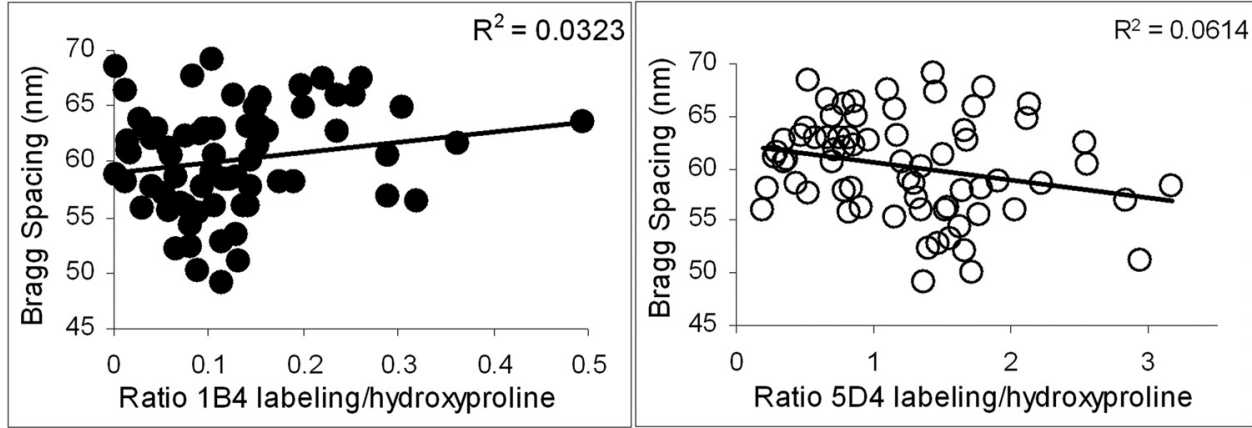

FIGURE 4. Correlation of sulfated KS antibody labeling (relative to $\mathrm{BAC}-\mathrm{ABC}$ core) per hydroxyproline in relation to collagen Bragg spacing for lesser (left, $1 \mathrm{~B} 4$ reactive) and more highly (right, 5D4 reactive) sulfated KS. 
acid; later, small leucine-rich PGs, particularly KS PGs most likely influence the water-binding characteristics of the stroma. Conrad et al., ${ }^{48}$ studying gene expression associated with chick corneal development, including those genes involved in endothelial transport, found 5- to 10-fold increases in sodiumpotassium ATPase transporter, sodium bicarbonate transporter, and carbonic anhydrase II (CA2), alongside increases in KS gene expression from E9 to E16. CA2 in particular, through increased transport of $\mathrm{Na}^{+} / \mathrm{HCO}_{3}{ }^{-}$and $\mathrm{Cl}^{-}$would be expected to drive thinning of the cornea. Perhaps the accumulation of oversulfated KS during this time serves to maintain local collagen hydration, as gross dehydration and compaction progress through the action of the nascent endothelial pump.

\section{References}

1. Linsenmayer TF, Fitch JM, Gordon MK, et al. Development and roles of collagenous matrices in the embryonic avian cornea. Prog Retin Eye Res. 1998;17:231-265.

2. Quantock AJ, Young RD. Development of the corneal stroma and the collagen-proteoglycan associations that help define its structure and function. Dev Dyn. 2008;237:2607-2621.

3. Birk DE, Trelstad RL. Extracellular compartments in matrix morphogenesis: collagen fibril, bundle, and lamellar formation by corneal fibroblasts. J Cell Biol. 1984;99:2024-2033.

4. Coulombre AJ, Coulombre JL. Corneal development, I: corneal transparency. J Cell Comp Physiol. 1958;51:1-11.

5. Hay ED, Revel J-P. Fine structure of the developing avian cornea. In: Wolsky A, Chen PS, eds. Monographs in Developmental Biology. Vol. 1. Basel, Switzerland: S. Karger; 1969:1-144.

6. Trelstad RL, Coulombre AJ. Morphogenesis of the collagenous stroma in the chick cornea. J Cell Biol. 1971;50:840-858.

7. Maurice DM. The structure and transparency of the cornea. J Physiol (Lond). 1957;186:263-286.

8. Benedek GB. Theory of transparency of the eye. Appl Opt. 1971; 10:459-473.

9. Borcherding MS, Blacik LJ, Sittig RA, Bizzell JW, Breen M, Weinstein HG. Proteoglycans and collagen fibre organization in human corneoscleral tissue. Exp Eye Res. 1975;21:59-70.

10. Blochberger TC, Vergnes JP, Hempel J, Hassell JR. cDNA to chick lumican (corneal keratan sulfate proteoglycan) reveals homology to the small interstitial proteoglycan gene family and expression in muscle and intestine. J Biol Chem. 1992;267:347-352.

11. Corpuz LM, Funderburgh JL, Funderburgh ML, Bottomley GS, Prakash S, Conrad GW. Molecular cloning and tissue distribution of keratocan: bovine corneal keratan sulfate proteoglycan 37A. J Biol Chem. 1996;271:9759-9763.

12. Funderburgh JL, Corpuz LM, Roth MR, Funderburgh ML, Tasheva ES, Conrad GW. Mimecan, the $25-\mathrm{kDa}$ corneal keratan sulfate proteoglycan, is a product of the gene producing osteoglycin. J Biol Chem. 1997;272:28089-28095.

13. Midura RJ, Hascall VC. Analysis of the proteoglycans synthesized by corneal explants from embryonic chicken, II: structural characterization of the keratan sulfate and dermatan sulfate proteoglycans from corneal stroma. J Biol Chem. 1989;264:1423-1430.

14. Funderburgh JL, Funderburgh ML, Mann MM, Conrad GW. Unique glycosylation of three keratan sulfate proteoglycan isoforms $J$ Biol Chem. 1991;266:14226-14231

15. Bettelheim FA, Plessy B. The hydration of proteoglycans of bovine cornea. Biochim Biophys Acta 1975;381:203-214.

16. Anseth A. Glycosaminoglycans in the developing corneal stroma. Exp Eye Res. 1961;1:116-121.

17. Hassell JR, Newsome DA, Krachmer JH, Rodrigues MM. Macular corneal dystrophy: failure to synthesize a mature keratan sulfate proteoglycan. Proc Natl Acad Sci USA. 1980;77:3705-3709.

18. Akama TO, Nishida K, Nakayama J, et al. Macular corneal dystrophy type I and type II are caused by distinct mutations in a new sulphotransferase gene. Nat Genet. 2000;26:237-241.

19. Chakravarti S, Magnuson T, Lass JH, Jepsen KJ, LaMantia C, Carroll $\mathrm{H}$. Lumican regulates collagen fibril assembly: Skin fragility and corneal opacity in the absence of lumican. J Cell Biol. 1998;141: $1277-1286$
20. Chakravarti S, Petroll WM, Hassell JR, et al. Corneal opacity in lumican-null mice: defects in collagen fibril structure and packing in the posterior stroma. Invest Ophthalmol Vis Sci. 2000;41:33653373.

21. Hayashida Y, Akama TO, Beecher N, et al. Matrix morphogenesis in cornea is mediated by the modification of keratan sulfate by GlcNAc 6-O sulfotransferase. Proc Natl Acad Sci USA. 2006;103: 13333-13338.

22. Funderburgh JL, Caterson B, Conrad GW. Keratan sulfate proteoglycan during embryonic development of the chicken cornea. Dev Biol. 1986;116:267-277.

23. Takahashi I, Nakamura Y, Hamada Y, Nakazawa K. Immunohistochemical analysis of proteoglycan biosynthesis during early development of the chicken cornea. J Biochem. 1999;126:804814.

24. Zhang Y, Conrad AH, Tasheva ES, et al. Detection and quantification of sulfated disaccharides from keratan sulfate and chondroitin/dermatan sulfate during chick corneal development by ESI-MS/MS. Invest Ophthalmol Vis Sci. 2005;46:1604-1614.

25. Young RD, Gealy EC, Liles M, Caterson B, Ralphs JR, Quantock AJ. Keratan sulfate glycosaminoglycan and the association with collagen fibrils in rudimentary lamellae in the developing avian cornea. Invest Ophthalmol Vis Sci. 2007;48:3083-3088.

26. Conrad AH, Conrad GW. The keratocan gene is expressed in both ocular and non-ocular tissues during early chick development. Matrix Biol. 2003;22:323-337.

27. Dunlevy JR, Beales MP, Berryhill BL, Cornuet PK, Hassell JR. Expression of the keratan sulfate proteoglycans lumican, keratocan and osteoglycin/mimecan during chick corneal development. Exp Eye Res. 2000;70:349-362.

28. Cornuet PK, Blochberger TC, Hassell JR. Molecular polymorphism of lumican during corneal development. Invest Ophthalmol Vis Sci. $1994 ; 35: 870-877$.

29. Cai CX, Gibney E, Gordon MK, Marchant JK, Birk DE, Linsenmayer TF. Characterization and developmental regulation of avian corneal $\beta$-1,4-galactosyltransferase mRNA. Exp Eye Res. 1996;63:193200.

30. Hart GW. Biosynthesis of glycosaminoglycans during corneal development. J Biol Chem. 1976;251:6513-6521.

31. Meek KM, Quantock AJ. The use of x-ray scattering techniques to determine corneal ultrastructure. Prog Retin Eye Res. 2001;20:95137 .

32. Blain EJ, Gilbert SJ, Hayes AJ, Duance VC. Disassembly of the vimentin cytoskeleton disrupts articular cartilage chondrocyte homeostasis. Matrix Biol. 2006;25:398-408.

33. Caterson B, Christner JE, Baker JR. Identification of a monoclonal antibody that specifically recognises corneal and skeletal keratan sulfate. J Biol Chem. 1983;258:8848-8854.

34. Caterson B, Christner JE, Baker JR, Couchman JR. Production and characterization of monoclonal antibodies directed against connective tissue proteoglycans. Fed Proc. 1985;44:386-393.

35. Mehmet H, Scudder P, Tang PW, Hounsell EF, Caterson B, Feizi T. The antigenic determinants recognized by three monoclonal antibodies to keratan sulfate involve hepta- or larger oligosaccharides of the poly (N-acetyllactosamine) series. Eur J Biochem. 1986;157: 385-391.

36. Young RD, Tudor D, Hayes AJ, et al. Atypical composition and ultrastructure of proteoglycans in the mouse corneal stroma. Invest Ophthalmol Vis Sci. 2005 46:1973-1978.

37. Coleman JR, Herrmann H, Bess BJ. Biosynthesis of collagen and non-collagen protein during development of the chick cornea. Cell Biol. 1965;25:69-78.

38. Conrad GW. Collagen and mucopolysaccharide biosynthesis in the developing chick cornea. Dev Biol. 1970;21(3):292-317.

39. Oeben M, Keller R, Stuhlsatz H, Greiling H. Constant and variable domains of different disaccharide structure in corneal keratan sulphate chains. Biochem J. 1987;248:85-93.

40. Akama TO, Misra AK, Hindsgaul O, Fukuda MN. Enzymatic synthesis in vitro of the disulfated disaccharide unit of corneal keratan sulfate. J Biol Chem. 2002;277:42505-42513.

41. Akama TO, Nakayama J, Nishida K, et al. Human corneal GlcNac 6-O-sulfotransferase and mouse intestinal GlcNac 6-O-sulfotrans- 
ferase both produce keratan sulfate. J Biol Chem. 2001;276: 16271-16278.

42. Conrad GW, Woo ML. Synthesis of 3'-phosphoadenosine-5'-phosphosulfate (PAPS) increases during corneal development. J Biol Chem. 1979;255:3086-3091.

43. Quantock AJ, Kinoshita S, Capel MS, Schanzlin DJ. A synchrotron $\mathrm{x}$-ray diffraction study of developing chick corneas. Biophys $J$. 1998;74:995-998.

44. Siegler V, Quantock AJ. Two-stage compaction of the secondary avian cornea during development. Exp Eye Res. 2002;74:427-431.
45. Hodson S, Miller F. The bicarbonate ion pump in the endothelium which regulates the hydration of rabbit cornea. J Physiol. 1976; 263:563-577.

46. Maurice DM. The location of the fluid pump in the cornea. J Physiol. 1972;221(1):43-54.

47. Davson H. The hydration of the cornea. J Biochem. 1955;59:24-28.

48. Conrad AH, Zhang Y, Walker AR, et al. Thyroxine affects expression of KS PG-related genes, the carbonic anhydrase II gene, and KS sulfation in the embryonic chicken cornea. Invest Ophthalmol Vis Sci. 2006;47:120-132. 